\title{
GENERALIZATIONS OF A WELL-KNOWN RESULT IN MATRIX THEORY
}

\author{
by R. C. THOMPSON \\ (Received 5 March, 1964)
}

Let $A$ and $C$ be $m \times m$ matrices and let $B$ and $D$ be $n \times n$ matrices, all with elements in a field $F$. Let $A^{T}$ denote the transpose of $A$. A well-known theorem states that, if every $m \times m$ matrix $X$ for which $A X=X A$ also satisfies $C X=X C$, then $C=\phi(A)$ for some polynomial $\phi(\lambda)$. In this note we establish the following simple generalizations.

THEOREM 1. Let $A$ and $B$ have the same minimal polynomial $m(\lambda)$. If each $m \times n$ matrix $X$ over $F$ for which $A X=X B$ also satisfies $C X=X D$, then $C=\phi(A)$ and $D=\phi(B)$ for a polynomial $\phi(\lambda)$ over $F$.

THEOREM 2. Let $n=m$. If each symmetric $m \times m$ matrix $X$ over $F$ for which $A X=X A^{T}$ also satisfies $C X=X D$, then $D^{T}=C=\phi(A)$ for a polynomial $\phi(\lambda)$ over $F$.

These results may be proved as easily as the classical result. It is possible to base the proofs on the Jordan canonical form under similarity by first extending $F$ to a field $K$ in which $m(\lambda)$ splits, next showing that the hypotheses will still be valid if $X$ is permitted to have elements in $K$, then transforming $A$ and $B$ to their Jordan forms and hence establishing the existence of $\phi(\lambda)$ over $K$, and finally showing that $\phi(\lambda)$ may be taken to have coefficients in $F$. We give proofs based on the rational canonical form under similarity.

Let $f(\lambda)=f_{r} \lambda^{r}+\ldots+f_{0}$ and $g(\lambda)=g_{s} \lambda^{s}+\ldots+g_{0}$ be two monic $\left(f_{r}=g_{s}=1\right)$ nonconstant polynomials. Let $C(f(\lambda))$ denote the companion matrix of $f(\lambda)$; it is defined in $[1, \mathrm{p} .148]$. A persymmetric matrix is one constant along each diagonal perpendicular to the main diagonal. Let $\bar{C}$ be a matrix with $r$ columns and let $\bar{D}$ be a matrix with $s$ rows.

Leмma. Each $r \times s$ matrix $X$ satisfying

$$
C(f(\lambda)) X=X C(g(\lambda))^{T}
$$

is persymmetric. If $f(\lambda)$ divides $g(\lambda)$ and if the first column of $\bar{C} X$ is zero for each $X$ over $F$ satisfying (1), then $\bar{C}=0$. If $g(\lambda)$ divides $f(\lambda)$ and if the first row of $X \bar{D}$ is zero for each $X$ over $F$ satisfying (1), then $\bar{D}=0$.

Proof. Let $X=\left(x_{i j}\right)$. Comparing the $(i, j)$ elements of the two sides of $(1)$ for $i<r$ and $j<s$, we have $x_{i+1, j}=x_{i, j+1}$. Hence $X$ is persymmetric and we may set $x_{i j}=x_{i+j-2}$ for $1 \leqq i \leqq r$ and $1 \leqq j \leqq s$. Let $g(\lambda) / f(\lambda)=h(\lambda)=h_{t} \lambda^{t}+\ldots+h_{0}$. For (1) to hold it is necessary and sufficient that

$$
\begin{gathered}
\sum_{\alpha=0}^{r} f_{\alpha} x_{\alpha+\beta}=0 \quad \text { for all } \beta \geqq 0 \text { and } \leqq s-2, \\
\sum_{\alpha=0}^{s} g_{\alpha} x_{\alpha+\beta}=0 \text { for all } \beta \geqq 0 \text { and } \leqq r-2, \\
\sum_{\alpha=0}^{s-r-1} g_{\alpha} x_{\alpha+r-1}+\sum_{\alpha=0}^{r-1}\left(g_{\alpha+s-r}-f_{\alpha}\right) x_{\alpha+s-1}=0 .
\end{gathered}
$$


If $r=1$ there are no equations (2b). Equation (2c) is an identity if $r=s$. The polynomial identity $g(\lambda)=f(\lambda) h(\lambda)$ implies relations among the $g_{i}, f_{i}, h_{i}$ which in turn imply that

$$
\sum_{\alpha=0}^{s} g_{\alpha} x_{\alpha+\beta}=\sum_{\mu=0}^{t} h_{\mu}\left(\sum_{\alpha=0}^{r} f_{\alpha} x_{\alpha+\mu+\beta}\right) \text { for all } \beta \geqq 0 \text { and } \leqq r-2 \text {. }
$$

Hence each equation ( $2 b)$ is a linear combination of some of equations $(2 a)$. Since $f(\lambda)$ also divides $g(\lambda)-\lambda^{s-r} f(\lambda)$, equation $(2 c)$ is, in a like manner, a linear combination of some of equations (2a). Hence (1) will hold if and only if (2a) is satisfied. From the form of (2a) it follows that $x_{0}, x_{1}, \ldots, x_{r-1}$ are independent variables with $x_{r}, \ldots, x_{r+s-2}$ determined in terms of these independent variables. Then, if $\bar{C}_{i}$ is column $i$ of $\bar{C}$, the first column of $\bar{C} X$ is $\bar{C}_{1} x_{0}+\ldots+\bar{C}_{r} x_{r-1}$, which can be zero for all choices of $x_{0}, \ldots, x_{r-1}$ in $F$ only if $\bar{C}=0$. The proof of the other case is similar.

We now give together the proofs of Theorems 1 and 2. Let $f_{1}(\lambda), f_{2}(\lambda), \ldots, f_{a}(\lambda)$ denote the nontrivial invariant factors of $\lambda I_{m}-A$ and let $g_{1}(\lambda), g_{2}(\lambda), \ldots, g_{b}(\lambda)$ denote the nontrivial invariant factors of $\lambda I_{n}-B$. Each $f_{i}(\lambda)$ and each $g_{i}(\lambda)$ divides the common minimal polynomial $m(\lambda)=f_{a}(\lambda)=g_{b}(\lambda)$ of $A$ and $B$.

In Theorem 2 we have $B=A^{T}, b=a$, and $g_{i}(\lambda)=f_{i}(\lambda)$ for all $i \leqq a$. Nonsingular matrices $S$ and $T$ exist over $F$ such that

and

$$
S A S^{-1}=C\left(f_{1}(\lambda)\right)+C\left(f_{2}(\lambda)\right) \dot{+} \ldots \dot{+} C\left(f_{a}(\lambda)\right)=A_{1}, \text { say, }
$$

$$
T B T^{-1}=C\left(g_{1}(\lambda)\right)^{T} \dot{+} C\left(g_{2}(\lambda)\right)^{T} \dot{+} \ldots \dot{+} C\left(g_{b}(\lambda)\right)^{T}=B_{1}, \text { say. }
$$

Here $\dot{+}$ denotes direct sum. For Theorem 2 take $T^{-1}=S^{T}$. Let $C_{1}=S C S^{-1}, X_{1}=S X T^{-1}$, $D_{1}=T D T^{-1}$. In Theorem $2, X_{1}$ is symmetric if and only if $X$ is. Then $A X=X B, A X=X A^{T}$, $C X=X D$ will hold if and only if, respectively, $A_{1} X_{1}=X_{1} B_{1}, A_{1} X_{1}=X_{1} A_{1}^{T}, C_{1} X_{1}=X_{1} D_{1}$. Partition the matrices $C_{1}, X_{1}, D_{1}$ into the forms $C_{1}=\left(C_{\alpha \beta}\right), X_{1}=\left(X_{\alpha \beta}\right), D_{1}=\left(D_{\alpha \beta}\right)$, where $C_{\alpha \beta}$ is (degree $\left.f_{\alpha}(\lambda)\right) \times\left(\right.$ degree $\left.f_{\beta}(\lambda)\right), X_{\alpha \beta}$ is (degree $\left.f_{\alpha}(\lambda)\right) \times\left(\right.$ degree $\left.g_{\beta}(\lambda)\right), D_{\alpha \beta}$ is (degree $\left.g_{\alpha}(\lambda)\right) \times$ (degree $g_{\beta}(\lambda)$ ).

For Theorem 1 set all $X_{\alpha \beta}$ equal to zero except for $X_{i b}$ for one fixed $i \leqq a$. Then $A_{1} X_{1}=X_{1} B_{1}$ will hold if $C\left(f_{l}(\lambda)\right) X_{i b}=X_{i b} C\left(g_{b}(\lambda)\right)^{T}$. From $C_{1} X_{1}=X_{1} D_{1}$ follows $C_{j i} X_{i b}=0$ if $j \neq i$. Hence $C_{j i}=0$. Next set all $X_{\alpha \beta}$ equal to zero except for $X_{a j}$ for one fixed $j \leqq b$. Then $A_{1} X_{1}=X_{1} B_{1}$ will hold if $C\left(f_{a}(\lambda)\right) X_{a j}=X_{a j} C\left(g_{j}(\lambda)\right)^{T}$. From $C_{1} X_{1}=X_{1} D_{1}$ follows $X_{a j} D_{j i}=0$ for $i \neq j$. Hence $D_{j i}=0$.

For Theorem 2 set all $X_{\alpha \beta}$ equal to zero except for $X_{j j}$ for one fixed $j \leqq a$. Then $A_{1} X_{1}=X_{1} A_{1}^{T}$ will hold if $C\left(f_{j}(\lambda)\right) X_{j j}=X_{j j} C\left(f_{j}(\lambda)\right)^{T}$. So $X_{j j}$ and therefore $X_{1}$ is symmetric. Then $C_{1} X_{1}=X_{1} D_{1}$ implies that $C_{i j} X_{j j}=0$ and $X_{j j} D_{j i}=0$ for $i \neq j$. Therefore $C_{i j}=0$ and $D_{j i}=0$. Thus, in both Theorems, $C_{1}$ and $D_{1}$ are block diagonal.

For the moment suppose that the first row of the last diagonal block $C_{a a}$ of $C_{1}$ is zero. Set all $X_{\alpha \beta}$ equal to zero except for $X_{a b}$. Then $A_{1} X_{1}=X_{1} B_{1}$ or $A_{1} X_{1}=X_{1} A_{1}^{T}$ will hold if $C\left(f_{a}(\lambda)\right) X_{a b}=X_{a b} C\left(g_{b}(\lambda)\right)^{T}$. Thus $X_{1}$ is symmetric in the case $B=A^{T}$. From $C_{1} X_{1}=X_{1} D_{1}$ we get $C_{a a} X_{a b}=X_{a b} D_{b b}$. Since the first row of $C_{a a} X_{a b}$ is zero, the first row of $X_{a b} D_{b b}$ is zero also; hence $D_{b b}=0$, and then $C_{a a}=0$. Next set all $X_{\alpha \beta}$ equal to zero except for $X_{i b}$ for one 
fixed $i<a$ and $X_{a j}$ for one fixed $j<b$. For Theorem 2 take $i=j$ and $X_{a i}=X_{i a}^{T}$ so as to make $X_{1}$ symmetric. Then $A_{1} X_{1}=X_{1} A_{1}^{T}$ will hold if $C\left(f_{i}(\lambda)\right) X_{i b}=X_{i b} C\left(g_{b}(\lambda)\right)^{T}$ and $A_{1} X_{1}=X_{1} B_{1}$ will hold if also $C\left(f_{a}(\lambda)\right) X_{a j}=X_{a j} C\left(g_{j}(\lambda)\right)^{T}$. Then $C_{1} X_{1}=X_{1} D_{1}$ yields $C_{i l} X_{i b}=0$ and $X_{a j} D_{j j}=0$ (because $C_{a a}=0$ and $D_{b b}=0$ ). Thus $C_{i i}=0$ and $D_{j j}=0$ and so both $C_{1}$ and $D_{1}$ are zero.

Now, for a fixed $i$ with $0 \leqq i<$ degree $m(\lambda)$, the first row of $\left[C\left(f_{a}(\lambda)\right)\right]^{i}$ is entirely zero except for a single 1 at column $i+1$. Thus it is possible to find a polynomial $\phi(\lambda)$ over $F$ such that the first row of $C_{a a}-\phi\left(C\left(f_{a}(\lambda)\right)\right.$ is entirely zero. It follows from $A_{1} X_{1}=X_{1} B_{1}$ that $A_{1}^{k} X_{1}=X_{1} B_{1}^{k}$ for $k=0,1,2, \ldots$; hence $\phi\left(A_{1}\right) X_{1}=X_{1} \phi\left(B_{1}\right)$, and therefore $\left(C_{1}-\phi\left(A_{1}\right)\right) X_{1}$ $=X_{1}\left(D_{1}-\phi\left(B_{1}\right)\right)$. Since the first row of the last diagonal block of $C_{1}-\phi\left(A_{1}\right)$ is zero, it now follows that $C_{1}-\phi\left(A_{1}\right)=0$ and $D_{1}-\phi\left(B_{1}\right)=0$. Thus $C=\phi(A)$ and $D=\phi(B)$ as required.

COROLlary. If each $X$ over $F$ for which $A X=X A$ also satisfies $C X=X D$ then $C=D=\phi(A)$. If $A$ is symmetric and each symmetric $X$ over $F$ for which $A X=X A$ also satisfies $C X=X D$, then $C=D=\phi(A)$ and so $C=D$ is symmetric.

The last statement follows because if $A=A^{T}$ then $A X=X A$ is the same as $A X=X A^{T}$ so $C=\phi(A)$ and $D=\phi\left(A^{T}\right)=\phi(A)=C$.

\section{REFERENCE}

1. S. Perlis, The theory of matrices (Cambridge, Mass., 1952).

THE University of British Columbia

(Now at the University of California, Santa Barbara) 